\title{
Ciclos de vida comparados de Aedes aegypti (Diptera, Culicidae) do semiárido da Paraíba
}

\author{
Francisco P. de Castro Jr. ${ }^{1,2}$, Walter F. S. Martins ${ }^{1}$, Mauricio L. de Lucena Filho ${ }^{1,3}$ \\ Raul P. de Almeida ${ }^{4} \&$ Eduardo B. Beserra ${ }^{1,5}$
}

\footnotetext{
. Depto de Biologia, Universidade Estadual da Paraíba, Campus Universitário I, Rua Juvêncio Arruda, s/n, Bodocongó, 58109-753 Campina Grande, PB. Autor correspondente: ebarbosa@uepb.edu.br Programa de Pós-Graduação em Ciências e Tecnologia Ambiental, UEPB.

Bolsista Iniciação Científica, CNPq.

4. Embrapa Algodão, Rua Osvaldo Cruz, 1143, Centenário, 58107-720 Campina Grande, PB
}

\begin{abstract}
Life cycles compared to Aedes aegypti (Diptera, Culicidae) in the semi-arid region of Paraíba. The present work aims at comparing the life cycle between samples from different populations of Aedes aegypti (Linnaeus, 1762) collected in ten municipalities in the semi-arid region of state of Paraíba, Brazil. The life cycles were studied under $26 \pm 2{ }^{\circ} \mathrm{C}$, a relative humidity of $60 \pm 10 \%$ and a $12 \mathrm{~h}$ photophase. The development period, egg viability, and larval and pupal survival, were evaluated daily, as well as the sex ratio, longevity, size, and fecundity of adults. We performed a cluster analysis, using a Euclidean distance matrix by the method of unweighted mean. The duration and viability for the egg, larval and pupal stages had, respectively, an average change of 3.7 to 4.7 days and $82.8 \%$ to $97.7 \%$, from 9.1 to 10.8 days and $91.2 \%$ to $99.2 \%$, and 2.1 to 2.5 days and $93.5 \%$ to $98.4 \%$. The wing length ranged from 5.13 to $5.34 \mathrm{~mm}$ for females and from 4.18 to $4.25 \mathrm{~mm}$ for males. The population of A. aegypti originated from Pedra Lavrada registered the lowest fecundity (153.6 eggs/female), while the highest fecundity (310.6 eggs/female) was observed in Campina Grande. Cluster analysis based on the similarity of biological data revealed the formation of two major distinct groups, where populations of $A$. aegypti from Serra Branca and Cuité show greater similarity to each other. Differences in biological cycles were observed among populations of $A$. aegypti, demonstrating the ability of this species to vary in its biology and adapt to different environmental conditions. This may increase the number of $A$. aegypti that stay in these areas, thus increasing the risk of the dengue virus.
\end{abstract}

KEYWORDS. Aedini, vector, dengue, biological cycle.

RESUMO. O presente trabalho teve como objetivo comparar os ciclos de vida entre amostras de populações de Aedes aegypti (Linnaeus, 1762) coletadas em dez municípios localizados no semiárido paraibano. Os ciclos de vida foram estudados a uma temperatura de $26 \pm 2^{\circ} \mathrm{C}$, umidade relativa de $60 \pm 10 \%$ e fotofase de 12 horas. Diariamente foram avaliados os períodos de desenvolvimentos e as viabilidades das fases de ovo, larva e pupa, bem como a razão sexual, longevidade, tamanho e fecundidade dos adultos. Foi realizada uma análise de agrupamento, utilizandose uma matriz de distância euclidiana através do método da média não-ponderada. As durações e viabilidades para as fases de ovo, larva e pupa apresentaram respectivamente, uma variação média de 3,7 a 4,7 dias e $82,8 \%$ a $97,7 \%, 9,1$ a 10,8 dias e $91,2 \%$ a $99,2 \%$ e de 2,1 a 2,5 dias e $93,5 \%$ a $98,4 \%$. O comprimento alar foi de 5,13 a $5,34 \mathrm{~mm}$ para as fêmeas e de 4,18 a 4,25 mm para os machos. A menor fecundidade (153,6 ovos/fêmea) ocorreu na população de $A$. aegypti oriunda de Pedra Lavrada, enquanto que a maior fecundidade (310,6 ovos/fêmea) foi constatada para A. aegypti de Campina Grande. A análise de agrupamento com base na similaridade dos dados biológicos revelou a formação de dois grandes grupos distintos, onde as populações de $A$. aegypti de Serra Branca e Cuité apresentam maior similaridade entre si. As diferenças de ciclos biológicos verificadas entre as populações de $A$. aegypti demonstra a capacidade dessa espécie de sofrer variações na sua biologia e se adaptar às diferentes condições ambientais, favorecendo a permanência deste inseto nessas áreas com aumento do risco de transmissão do vírus da dengue.

PALAVRAS-CHAVE. Aedini, vetor, dengue, ciclo biológico.

Aedes aegypti (Linnaeus, 1762) é uma importante praga urbana, vetor do vírus que causa a dengue nas Américas e da febre amarela na América Central e do Sul e no oeste da África (Сноосноте et al., 2004; FANTinatTi et al., 2007). É um inseto amplamente distribuído principalmente em regiões com infraestrutura deficiente, que favorecem o desenvolvimento e o estabelecimento deste vetor e, consequentemente, dos sorotipos virais por ele veiculados (BESERRA et al., 2010).

OEstado da Paraíba apresenta condições climáticas e de falta de infraestrutura que favorecem a ocorrência do $A$. aegypti e da dengue. De acordo com o relatório de situação da dengue para a Paraíba, elaborado pelo Sistema Nacional de Vigilância em Saúde (Ministério DA SAÚDE, 2011), em 2010 foram notificados 6.667 casos, um incremento de $601,1 \%$ em relação ao mesmo período de 2009. Em 2012 foram registrados no estado 11.502 casos, com redução de $29,84 \%$ se comparado ao período de 2011 (16.396 notificações, SECRETARIA DE Saúde do Estado da Paraíba, 2013).

A ocorrência da dengue está vinculada à biologia e à ecologia do seu vetor e sua associação com o homem (NATAL, 2002). Na fase larval, esta associação se dá devido à necessidade de depósitos de água adequados ao seu desenvolvimento; na fase adulta, a associação com o homem ocorre como consequência do seu comportamento reprodutivo e da alimentação sanguínea, e seus efeitos trazem implicações para a transmissão da dengue. $O$ entendimento sobre esses aspectos bioecológicos do vetor é de grande importância epidemiológica, já que estudos dessa natureza geram informações sobre a dinâmica reprodutiva, hematofágica e da sua competência vetorial, e ajudam a entender o mecanismo de transmissão do vírus da dengue.

A maioria dos estudos que aborda o ciclo de vida de Aedes aegypti foi realizado avaliando-se populações isoladas e poucas contribuições mencionam comparações entre ciclos de populações de diferentes regiões geográficas. O ciclo de vida do A. aegypti apresenta padrões de crescimento, fecundidade e longevidade inerentes a cada população, que são influenciados por fatores intrínsecos à espécie e por fatores ambientais, 
próprios de cada região de origem do vetor (BESERRA et al., 2006; BeSERRA \& CASTRo JR., 2008; BeserRa et al., 2009a,b).

A ocorrência de variações interpopulacionais com relação às variáveis biológicas do vetor, como duração dos ciclos de vida e gonotrófico, sobrevivência, longevidade, tamanho efecundidade das fêmease duração do período de incubação extrínseco podem influenciar a capacidade vetorial do inseto, e consequentemente a dinâmica de transmissão do vírus em diferentes populações. A caracterização destes aspectos biológicos pode, ainda, ser utilizada em modelos de previsão de riscos da doença, que são ferramentas importantes no desenvolvimento de estratégias de controle do vetor (Maciel-de-Freitas et al., 2008). O objetivo deste trabalho foi comparar os padrões de desenvolvimento entre amostras de populações de $A$. aegypti coletadas em diferentes regiões do semiárido paraibano.

\section{MATERIAL E MÉTODOS}

As colônias das amostras das populações de Aedes aegypti e os bioensaios foram conduzidos no Laboratório de Entomologia do Núcleo de Sistemática e Bioecologia de Insetos da Universidade Estadual da Paraíba - UEPB, em sala de criação climatizada com temperatura de $26 \pm 2{ }^{\circ} \mathrm{C}$, umidade relativa de $60 \pm 10 \%$ e fotofase de 12 horas.

As amostras das populações foram obtidas a partir de ovos coletados em armadilhas de oviposição instaladas em municípios de duas mesorregiões da Paraíba, Agreste Paraibano e Mesorregião da Borborema. Na Mesorregião do Agreste Paraibano, as armadilhas foram instaladas nos municípios de Campina Grande (07 $13^{\prime} 50^{\prime} \mathrm{S}$, $\left.35^{\circ} 52^{\prime} 52^{\prime \prime} \mathrm{W}\right)$ e Queimadas ( $07^{\circ} 21^{\prime} 30^{\prime}$ 'S, 35 $\left.54^{\prime} 54^{\prime \prime} \mathrm{W}\right)$, localizados na Microrregião de Campina Grande, e Cuité (06 $\left.29^{\prime} 01^{\prime \prime} \mathrm{S}, 36^{\circ} 09^{\prime} 13^{\prime \prime} \mathrm{W}\right)$, na Microrregião do Curimataú Ocidental. Na Messorregião da Borborema as coletas ocorreram nos municípios de Livramento $\left(07^{\circ} 22^{\prime} 27^{\prime \prime} \mathrm{S}, 36^{\circ} 56^{\prime} 47^{\prime \prime} \mathrm{W}\right)$, Serra Branca $\left(07^{\circ} 29^{\prime} 00^{\prime}\right.$ 'S,

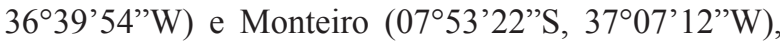
localizados na microrregião do Cariri Ocidental;

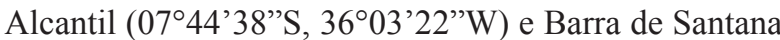
(07³1'13”S, 35'59'59”W) na Microrregião do Cariri Oriental; Pedra Lavrada $\left(06^{\circ} 45^{\prime} 28^{\prime \prime} \mathrm{S}, 36^{\circ} 28^{\prime} 15^{\prime \prime} \mathrm{W}\right)$ localizado no Seridó Oriental e Santa Luzia ( $06^{\circ} 52^{\prime} 20^{\prime}$ 'S, $36^{\circ} 55^{\prime} 07^{\prime}$ 'W), no Seridó Ocidental (Fig. 1).

Segundo o boletim epidemiológico $\mathrm{n}^{\circ} 05$ de $01 \mathrm{de}$ janeiro a 30 de março de 2013 da Secretaria Estadual de Saúde/PB, o município de Santa Luzia foi considerado de alto risco para dengue [Índice de Infestação Predial (IIP) $>3,9 \%$ e Taxa de Incidência $>60 / 100.000$ habitantes]; Cuité, Barra de Santana e Monteiro de médio risco (IIP $<3,9 \%$ e Taxa de Incidência de 20 a 60/100.000 habitantes); Campina Grande e Queimadas de baixo risco (IIP $<1 \%$ e Taxa de Incidência $<20 / 100.000$ habitantes); e Alcantil, Livramento e Serra Branca sem registros de casos de dengue.
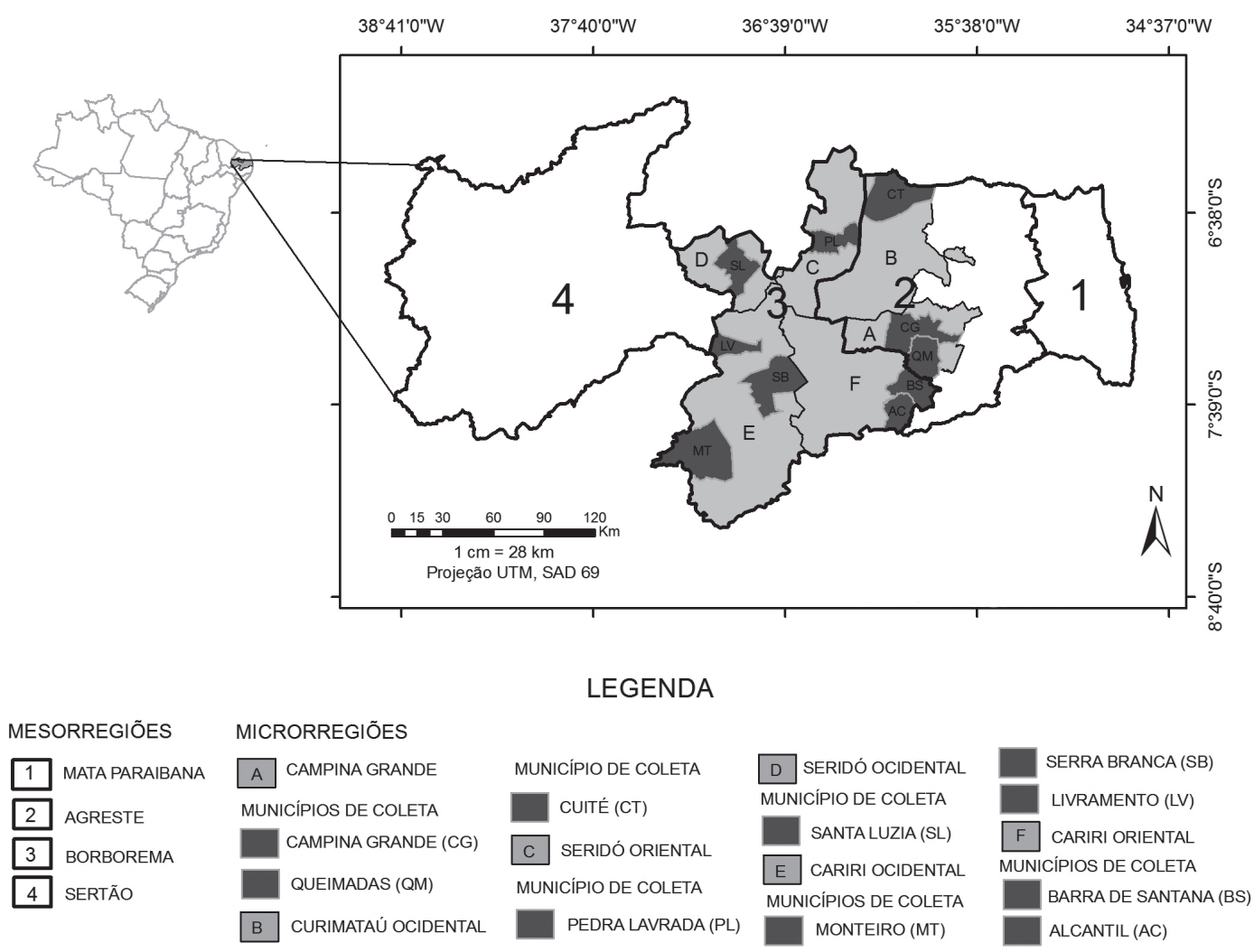

Fig. 1. Locais de coleta de Aedes aegypti nas Meso- e Microrregiões do Estado da Paraíba. 
Os ovos foram levados ao laboratório e acondicionados em bandejas plásticas (40 x $27 \times 7,5$ $\mathrm{cm}$ ) contendo água desclorada. Após a eclosão, ofertouse às larvas ração para peixe ornamental até atingirem o estágio de pupa. As pupas foram acondicionadas em copos descartáveis de $250 \mathrm{ml}$ com água desclorada e colocados em gaiolas de manutenção dos adultos $(40 \mathrm{~cm}$ x $40 \mathrm{~cm}$ x $30 \mathrm{~cm}$ ). Os adultos foram alimentados com solução de mel a $20 \%$ e às fêmeas permitido o repasto sanguíneo em codornas (Coturnix japonica) durante 15 minutos, três vezes por semana. Após o repasto, os ovos foram coletados em um funil plástico revestido por um papel filtro dentro de um copo com água, que serviu de substrato para a oviposição (BESERRA \& CASTRO JR., 2008; BESERRA et al., 2009a,b).

Os ciclos de vida foram estudados a partir da primeira geração de laboratório $(\mathrm{F})$, seguindo-se o delineamento experimental inteiramente ao acaso com dez tratamentos (populações) e quatro repetições. Diariamente foram mensuradas as temperaturas das águas de desenvolvimento larval e pupal com utilização de termômetro químico com aferição até $60^{\circ} \mathrm{C}$ e $\mathrm{pH}$ por meio de um $\mathrm{pH}$-metro digital.

A avaliação do período embrionário foi conduzida a partir das 20 primeiras posturas, distribuídas em placas de Petri de 9,0 cm de diâmetro x 1,5 cm de profundidade, contendo água destilada suficiente para encobrir os ovos. Foram realizadas avaliações diárias, registrandose o período de desenvolvimento e a mortalidade das fases de ovo, larva e pupa, a razão sexual, a longevidade, o número de ovos por fêmea e o tamanho dos adultos. Para a verificação do tamanho dos adultos, utilizouse como parâmetro a envergadura das asas, que foram mensuradas com ocular micrométrica acoplada ao microscópio estereoscópico; a medida foi tomada da álula até a extremidade da asa, desconsiderando-se a franja (GAma et al., 2005; Mohammed \& ChadeE, 2011).

As variáveis biológicas foram submetidas à análise de variância e as médias comparadas pelo teste de Tukey $(\mathrm{P} \leq 0,05)$. Com esses dados foi realizada uma análise de agrupamento (“Cluster analyses”), utilizandose uma matriz de distância euclidiana através do método da média não-ponderada, para se avaliar o grau de similaridade entre as populações estudadas (BESERRA et al., 2003).

\section{RESULTADOS E DISCUSSÃO}

Ciclos de vida comparados. A temperatura média das águas onde se desenvolveram os indivíduos de $A$. aegypti apresentou poucas variações. A menor temperatura $\left(24,7^{\circ} \mathrm{C}\right)$ ocorreu durante o desenvolvimento de larvas provenientes do município de Monteiro e a maior $\left(25,1^{\circ} \mathrm{C}\right)$ para aquelas de Livramento e Alcantil (Tab. I). Nestas condições, não se constatou diferenças significativas com relação à duração e viabilidade das fases de ovo, larva e pupa entre as amostras dos diferentes municípios (Tabs I, II). Tal similaridade não foi verificada por BESERRA \& CASTRO JR. (2008) e BESERRA et al. (2009a,b), que demonstraram que o padrão de desenvolvimento para as fases de ovo, larva e pupa apresenta variações interpopulacionais de acordo com a região de origem do vetor.

Quando se analisou os valores médios obtidos, verificou-se que para a fase de ovo o tempo de desenvolvimento está de acordo com os descritos por BesErRA et al. (2006) (média de 4,1 a 4,7 dias) e por Beserra \& CASTro JR. (2008) (média de 3,8 a 4,4 dias). No entanto, estudos realizados por BESERRA et al. (2009b) com amostras de populações do vetor de Boqueirão, Campina Grande e Remígio, na Paraíba, constaram que, a $26^{\circ} \mathrm{C}$, a duração média desta fase foi de $6,1,4,7$ e 5,2 dias respectivamente, portanto maiores que as encontradas no presente estudo.

Para a fase larval, o período de desenvolvimento foi considerado longo ( 9,1 a 10,8 dias) se confrontado à duração média observada para indivíduos de $A$. aegypti coletados em outros municípios da Paraíba como Boqueirão (5,6 dias) e Remígio (5,2 dias) (BESERRA et al., 2009b), Brejo dos Santos (6,8 dias), Boqueirão (6,8 dias), Itaporanga (8,3 dias) e Remígio (6,3 dias) (BESERRA

Tab. I. Duração (dias) ( $\bar{X} \pm$ EP) das fases de ovo, larva e pupa, e razão sexual das populações de Aedes aegypti (Linnaeus, 1762) de diferentes regiões do semiárido paraibano. Temperatura ambiente: $26 \pm 2,0^{\circ} \mathrm{C}$, UR: $60 \pm 10 \%$ e fotofase: 12 horas [ ${ }^{1}$, Médias originais. Para efeito da análise estatística os dados foram transformados em; $\sqrt{X+0,50}$, não significativo pelo teste $\mathrm{F}(\mathrm{P} \leq 0,05)]$.

\begin{tabular}{|c|c|c|c|c|c|}
\hline \multirow{2}{*}{ População } & \multirow{2}{*}{ Temperatura da água $\left({ }^{\circ} \mathrm{C}\right)$} & \multicolumn{3}{|c|}{ Duração } & \multirow{2}{*}{ Razão sexual ${ }^{1,2}$} \\
\hline & & $\mathrm{Ovo}^{1,2}$ & Larva $^{2}$ & Pupa $^{2}$ & \\
\hline Campina Grande & $24,98 \pm 0,14$ & $4,59 \pm 0,33$ & $9,35 \pm 0,60$ & $2,47 \pm 0,10$ & $0,31 \pm 0,09$ \\
\hline Pedra Lavrada & $24,94 \pm 0,11$ & $4,79 \pm 0,31$ & $9,96 \pm 0,49$ & $2,34 \pm 0,02$ & $0,32 \pm 0,02$ \\
\hline Santa Luzia & $25,08 \pm 0,06$ & $3,87 \pm 0,17$ & $9,84 \pm 0,29$ & $2,36 \pm 0,20$ & $0,45 \pm 0,10$ \\
\hline Livramento & $25,14 \pm 0,10$ & $3,79 \pm 0,38$ & $10,57 \pm 0,42$ & $2,41 \pm 0,07$ & $0,41 \pm 0,05$ \\
\hline Queimadas & $25,08 \pm 0,09$ & $4,33 \pm 0,29$ & $10,82 \pm 0,26$ & $2,44 \pm 0,05$ & $0,47 \pm 0,05$ \\
\hline Alcantil & $25,14 \pm 0,12$ & $4,29 \pm 0,20$ & $9,28 \pm 0,60$ & $2,18 \pm 0,14$ & $0,39 \pm 0,03$ \\
\hline Serra Branca & $24,91 \pm 0,16$ & $4,34 \pm 0,21$ & $10,25 \pm 1,08$ & $2,41 \pm 0,06$ & $0,42 \pm 0,05$ \\
\hline Barra de Santana & $25,04 \pm 0,11$ & $4,65 \pm 0,31$ & $9,15 \pm 0,94$ & $2,59 \pm 0,09$ & $0,48 \pm 0,06$ \\
\hline Monteiro & $24,75 \pm 0,13$ & $4,07 \pm 0,22$ & $10,89 \pm 0,30$ & $2,43 \pm 0,10$ & $0,51 \pm 0,03$ \\
\hline Cuité & $24,83 \pm 0,11$ & $4,02 \pm 0,28$ & $10,29 \pm 0,45$ & $2,47 \pm 0,04$ & $0,42 \pm 0,02$ \\
\hline C.V. (\%) & - & 14,08 & 12,04 & 8,55 & 6,22 \\
\hline
\end{tabular}


Tab. II. Viabilidade (\%) ( $\bar{X} \pm$ EP) das fases de ovo, larva e pupa das populações de Aedes aegypti (Linnaeus, 1762) de diferentes regiões do semiárido paraibano. Temperatura ambiente: $26 \pm 2,0^{\circ} \mathrm{C}, \mathrm{UR}: 60 \pm 10 \%$ e fotofase: 12 horas [ ${ }^{1}$, não significativo pelo teste $\left.\mathrm{F}(\mathrm{P} \leq 0,05)\right]$.

\begin{tabular}{lcccc}
\hline \multirow{2}{*}{ População } & $\begin{array}{c}\text { Temperatura } \\
\text { da água }\left({ }^{\circ} \mathrm{C}\right)\end{array}$ & Ovo & Viabilidade \\
\cline { 3 - 4 } & $24,98 \pm 0,14$ & $92,60 \pm 4,96$ & Larva & Pupa \\
\hline Campina Grande & $24,94 \pm 0,11$ & $96,86 \pm 1,76$ & $91,25 \pm 3,20$ & $95,03 \pm 1,53$ \\
Pedra Lavrada & $25,08 \pm 0,06$ & $97,74 \pm 1,31$ & $95,25 \pm 3,54$ & $94,20 \pm 2,40$ \\
Santa Luzia & $25,14 \pm 0,10$ & $82,88 \pm 8,10$ & $92,75 \pm 7,25$ & $96,22 \pm 1,69$ \\
Livramento & $25,08 \pm 0,09$ & $86,22 \pm 6,17$ & $96,50 \pm 2,60$ & $98,46 \pm 0,90$ \\
Queimadas & $25,14 \pm 0,12$ & $93,35 \pm 2,53$ & $98,75 \pm 1,25$ & $97,43 \pm 0,82$ \\
Alcantil & $24,91 \pm 0,16$ & $96,68 \pm 1,68$ & $94,25 \pm 3,38$ & $96,41 \pm 1,21$ \\
Serra Branca & $25,04 \pm 0,11$ & $97,51 \pm 1,22$ & $99,25 \pm 0,75$ & $94,68 \pm 3,77$ \\
Barra de Santana & $24,75 \pm 0,13$ & $95,06 \pm 2,31$ & $93,25 \pm 5,76$ & $95,84 \pm 2,41$ \\
Monteiro & $24,83 \pm 0,11$ & $87,04 \pm 5,66$ & $98,75 \pm 1,25$ & $93,57 \pm 3,17$ \\
Cuité & - & 20,63 & $97,25 \pm 0,95$ & $97,44 \pm 0,64$ \\
\hline C.V. (\%) & & 7,57 & 4,39 \\
\hline
\end{tabular}

\& CASTRO JR., 2008). Neste último caso, a diferença pode ser explicada devido às variações nas densidades nas quais as larvas foram submetidas. No estudo de BESERRA \& CASTRO JR. (2008), as larvas estavam em menor densidade populacional (5 larvas $/ 250 \mathrm{ml} \mathrm{de}$ água), em relação a este estudo (100 larvas/1.000 $\mathrm{ml}$ de água). Essa maior densidade aumentou a competição por alimento e espaço interferindo no desenvolvimento larval do vetor, corroborando a afirmação de BESERRA et $a l$. (2009a) de que o aumento na densidade é prejudicial ao desenvolvimento larval de $A$. aegypti em função da disponibilidade de alimento. Segundo estes autores, a duração do desenvolvimento larval aumentou de 8,3 dias na densidade de 200 larvas/L, para 37,9 dias na densidade de 1250 larva/L quando foram ofertadas 100 $\mathrm{g}$ de ração por bandeja de criação.

Para a fase de pupa, as médias de desenvolvimento estão dentro da variação registrada por BESERRA et al. (2006) (2,1 a 2,7 dias) e por BeserRA \& CASTRo JR. (2008) ( 2,0 a 2,5 dias). A razão sexual teve variação média de 0,31 a 0,51 , constatando-se que, com exceção da população proveniente de Monteiro ( $\mathrm{rs}=0,51)$, houve, proporcionalmente, maior quantidade de machos do que fêmeas para as demais populações (Tab. I).

A viabilidade do estágio de ovo, larva e pupa foi considerada alta e está próxima das relatadas por SiLVA \& Silva (1999), BESERRA et al. (2006) e FARNESI et al. (2009), que foi acima de $80 \%$ (Tab. II). TeJERINA et al. (2009) estudaram quatro subpopulações sob condições seminaturais da província de Misiones (Argentina), provenientes de Posadas, San Javier, Bernardo de Irigoyen e Puerto Libertad, e também registraram altas porcentagens de sobrevivência larval e pupal $(97 \%$ a $100 \%)$.

Em todas as amostras do semiárido paraibano as fêmeas de $A$. aegypti foram significativamente mais longevas que os machos (Tab. III), o que também foi relatado para essa espécie por BESERRA et al. (2009a), e para outras espécies de Aedes, como Aedes albopictus Skuse, 1894 (CAlado \& Navarro-Silva, 2002; Lowenberg Neto \& Navarro-Silva, 2004; Delatte et al., 2009).

O número de ovos por fêmea diferiu significativamente de acordo com o município de coleta do vetor (Tab. III). A menor fecundidade (153,61 ovos/ fêmea) ocorreu na população de Pedra Lavrada, enquanto a de Campina Grande apresentou a maior fecundidade (310,61 ovos/fêmea). Com exceção de Campina Grande, Alcantil, Barra de Santana e Monteiro, onde o número médio de ovos por fêmea foi superior a 200, verificou-se

Tab. III. Longevidade dos adultos $(\bar{X} \pm \mathrm{EP})$ e número de ovos por fêmea $(\bar{X} \pm \mathrm{EP})$ de Aedes aegypti (Linnaeus, 1762$)$ de populações do semiárido paraibano. Temperatura ambiente: $26 \pm 2,0^{\circ} \mathrm{C}$, UR: $60 \pm 10 \%$ e fotofase: 12 horas $\left[{ }^{1}\right.$, Médias seguidas de mesma letra nas colunas não diferem entre si pelo teste de Tukey $(\mathrm{P} \leq 0,05) ;{ }^{2}$, Médias originais. Para efeito da análise estatística os dados foram transformados em $\sqrt{X+0,50}$ ].

\begin{tabular}{lccc}
\hline \multirow{2}{*}{ População } & \multicolumn{2}{c}{ Longevidade $^{1}$} & \multirow{2}{*}{ Número de ovos/fêmea $^{1,2}$} \\
\cline { 2 - 3 } & \multicolumn{1}{c}{ Macho } & Fêmea & $310,61 \pm 38,33 \mathrm{~A}$ \\
Campina Grande & $49,62 \pm 2,38 \mathrm{AB}$ & $71,47 \pm 3,27 \mathrm{AB}$ & $153,61 \pm 11,60 \mathrm{D}$ \\
Pedra Lavrada & $49,36 \pm 2,86 \mathrm{AB}$ & $62,69 \pm 4,01 \mathrm{ABC}$ & $155,22 \pm 5,55 \mathrm{CD}$ \\
Santa Luzia & $46,66 \pm 3,17 \mathrm{AB}$ & $51,32 \pm 3,3 \mathrm{CD}$ & $193,38 \pm 12,56 \mathrm{BCD}$ \\
Livramento & $37,82 \pm 0,96 \mathrm{~B}$ & $40,03 \pm 0,61 \mathrm{D}$ & $173,15 \pm 9,58 \mathrm{CD}$ \\
Queimadas & $40,82 \pm 2,24 \mathrm{~B}$ & $44,32 \pm 2,20 \mathrm{CD}$ & $298,47 \pm 31,50 \mathrm{AB}$ \\
Alcantil & $58,29 \pm 2,02 \mathrm{~A}$ & $73,07 \pm 4,12 \mathrm{~A}$ & $176,58 \pm 10,09 \mathrm{CD}$ \\
Serra Branca & $47,09 \pm 250 \mathrm{AB}$ & $62,48 \pm 2,54 \mathrm{ABC}$ & $265,14 \pm 50,09 \mathrm{ABC}$ \\
Barra de Santana & $42,92 \pm 2,60 \mathrm{~B}$ & $55,26 \pm 5,64 \mathrm{ABCD}$ & $228,92 \pm 16,57 \mathrm{ABCD}$ \\
Monteiro & $44,90 \pm 5,52 \mathrm{AB}$ & $53,92 \pm 6,48 \mathrm{BCD}$ & $180,24 \pm 5,17 \mathrm{CD}$ \\
Cuité & $51,00 \pm 4,29 \mathrm{AB}$ & $60,39 \pm 4,20 \mathrm{ABC}$ & 15,45 \\
C.V. (\%) & 6,53 & 14,78 & 15,45 \\
\hline
\end{tabular}


que a fecundidade média para os demais municípios foi menor do que as registradas por BESERRA et al. (2006), Beserra \& CASTro JR. (2008) e Beserra et al. (2009b); estes últimos observam uma média de 610,6 ovos/fêmea para A. aegypti em Boqueirão.

As diferenças nas variáveis biológicas aqui avaliadas e nos demais estudos mostram que o $A$. aegypti apresenta grande plasticidade fenotípica. Ou seja, mesmo submetendo-se distintas amostras populacionais às mesmas condições de laboratório, seus padrões de desenvolvimento sofreram variações, que podem estar associadas, além da região de origem do vetor, ao período e à estação de coleta, à geração do vetor em campo e às diferenças genotípicas interpopulacionais.

Esse fato reveste-se de grande importância já que quanto maior for a sua plasticidade genotípica e fenotípica, e quanto mais variado for o ciclo de vida do vetor, maior será sua capacidade de se adaptar às diferentes condições ambientais.

Comprimento das asas (mm). Não houve interação significativa entre sexo e população quanto ao comprimento das asas, porém, detectou-se diferença entre os sexos: as fêmeas $(5,13$ a 5,34 mm) foram maiores que os machos $(4,18$ a 4,25 mm), independentes da população. Essas diferenças também foram observadas em amostras de populações da Paraíba por BESERRA et al. (2009a) (fêmeas, 3,8 a 5,2 mm; machos, 3,4 a 4,2 $\mathrm{mm}$ ), e por Gama et al. (2005) (fêmeas, 2,45 a 2,91 mm; machos, 1,99 a 2,26 mm), embora neste caso os valores sejam menores aos aqui apresentados.

A massa pupal e a envergadura das asas são indicadores de fecundidade em A. albopictus e Aedes geniculatus (Olivier, 1791) (BLACKMORE \& LORD, 2000; Armbruster \& Hutchinson, 2002). Portanto, há maior fecundidade quanto maior for o comprimento alar e, consequentemente, a fêmea (BLACKMORE \& LORD, 2000); esta relação também é verificada nos machos. PonlawAT \& HARRINGTON (2007) registraram maior quantidade de espermatozóides nos testículos e em vesículas seminais de machos de A. aegypti que apresentavam tamanho corporal maior, indicando assim uma maior capacidade reprodutiva. Ressalta-se que o maior tamanho desses insetos é um indicativo de melhor capacidade de adaptação às mudanças nas condições ambientais e de maior competência vetorial (VARGAS et al., 2010; Mohammed \& CHADEe, 2011).

Análise de agrupamento das características biológicas de populações de Aedes aegypti. Com base na similaridade dos dados biológicos, verificou-se que foram formados dois grupos distintos (Fig. 2): um formado pelas populações de Campina Grande, Alcantil, Barra de Santana e Monteiro e o outro pelas de Pedra Lavrada, Santa Luzia, Livramento, Queimadas, Serra Branca e Cuité. Dentro do primeiro grupo, maiores similaridades foram observadas entre as amostras de populações de $A$. aegypti de Campina Grande e Alcantil, que constituíram um primeiro subgrupo, e de Barra de Santana e Monteiro, constituindo um segundo subgrupo. Em relação ao segundo grupo, foram formados três subgrupos: o primeiro formado por Pedra Lavrada e Santa Luzia, o segundo por Serra Branca e Cuité, esses dois subgrupos com maior similaridade entre suas populações, se comparados ao terceiro subgrupo, formado por amostras do vetor de Livramento e Queimadas (Fig. 2).

Deve-se ressaltar que em todos os subgrupos nenhuma das amostras das populações pertenciam a municípios de uma mesma microrregião, principalmente os subgrupos formados por Campina Grande e Alcantil, Serra Branca e Cuité, e Livramento e Queimadas, que foram constituídos de amostras provenientes de municípios de mesorregiões distintas (Fig. 1).

Durante o ciclo aquático do vetor (ovo, larva e pupa), as populações apresentaram grande semelhança interpopulacional com relação aos padrões de desenvolvimento, não se constatando diferenças

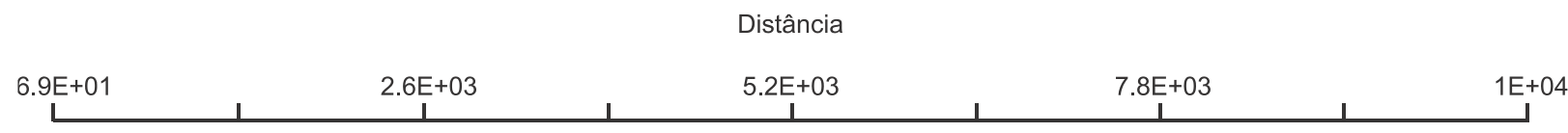

Informação residual (\%)

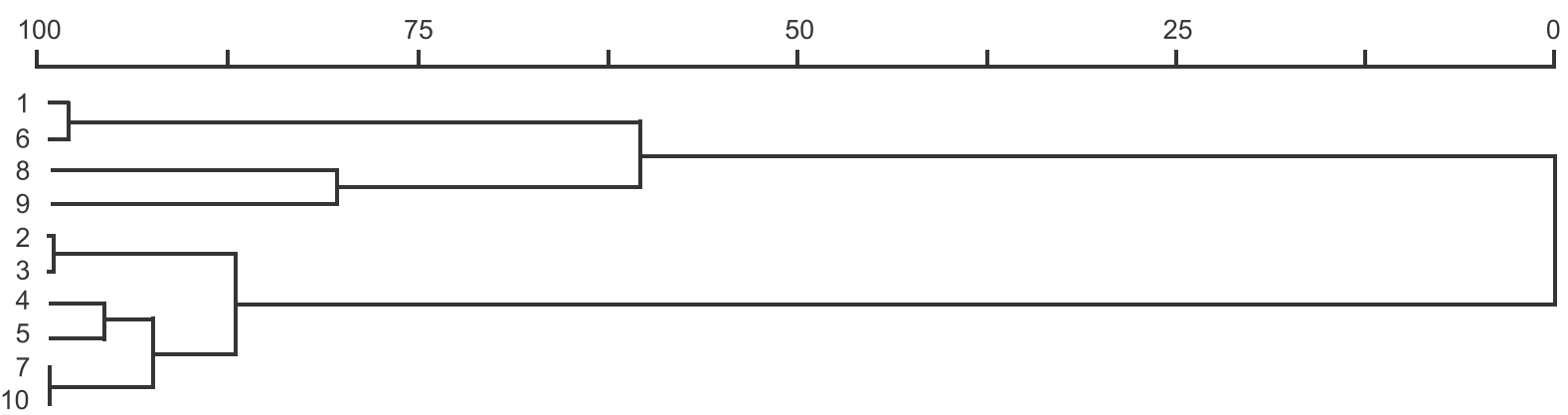

Fig. 2. Fenograma da análise de agrupamento de características biológicas de amostras de dez populações de Aedes aegypti do semiárido paraibano.1, Campina Grande; 2, Pedra Lavrada; 3, Santa Luzia; 4, Livramento; 5, Queimadas; 6, Alcantil; 7, Serra Branca; 8, Barra de Santana; 9, Monteiro; 10, Cuité. 
significativas entre as variáveis avaliadas. Contudo, na fase adulta, essas populações demonstraram maior variação nos padrões de longevidade e fecundidade, com destaque para as fêmeas de Campina Grande e Alcantil, que foram as mais longevas e de maior fecundidade em relação às demais. Assumindo que maior longevidade $\mathrm{e}$ fecundidade reflete em maior capacidade vetorial (JoY et al., 2010), pode-se considerar que as populações de Campina Grande e Alcantil são as que apresentam maior potencial para a disseminação do vírus da dengue. Porém, com base nos padrões de ciclos biológicos aqui verificados, essa espécie demonstrou a capacidade de sofrer variações na sua biologia e de se adaptar às diferentes condições ambientais. Tal capacidade pode favorecer uma maior permanência de $A$. aegypti nessas áreas e, consequentemente, aumentar o risco de transmissão do vírus da dengue.

Agradecimentos. As secretarias municipais de saúde dos municípios que integraram a pesquisa. Ao Programa de PósGraduação em Ciências e Tecnologia Ambiental e ao programa de incentivo a pesquisa, PROPESQ (Universidade Estadual da Paraíba), pelo financiamento da pesquisa. A Otávia Karla dos S. Apolinário, pela elaboração do mapa.

\section{REFERÊNCIAS BIBLIOGRÁFICAS}

Armbruster, P. \& Hutchinson, R. A. 2002. Pupal mass and wing length as indicators of fecundity in Aedes albopictus and Aedes geniculatus (Diptera: Culicidae). Journal of Medical Entomology 39:699-704.

Beserra, E. B. \& CASTro JR., F. P. 2008. Biologia comparada de populações de Aedes (Stegomyia) aegypti (L.) (Diptera: Culicidae) da Paraíba. Neotropical Entomology 37(1):81-85.

Beserra, E. B.; Castro JR., F. P.; Santos, J. W. dos; Santos, T. da S. \& Fernandes, C. R. M. 2006. Biologia e exigências térmicas de Aedes aegypti (Diptera: Culicidae) provenientes de quatro regiões bioclimáticas da Paraíba. Neotropical Entomology 35(6):853860.

Beserra, E. B.; Fernandes, C. R. M. \& Ribeiro, P. S. 2009a. Relação entre densidade larval e ciclo de vida, tamanho e fecundidade de Aedes (Stegomyia) aegypti (L.) (Diptera: Culicidae) em laboratório. Neotropical Entomology 38(6):847-852.

Beserra, E. B.; Fernandes, C. R. M.; Silva, S. A. de O.; Silva, L. A. DA \& SANTOS, J. W. DOS. 2009b. Efeitos da temperatura no ciclo de vida, exigências térmicas e estimativas do número de gerações anuais de Aedes aegypti (Diptera: Culicidae). Iheringia, Série Zoologia 99(2):142-148.

Beserra, E. B.; Fernandes, C. R. M.; Sousa, J. T. de; Freitas, E. M. DE \& SANTOS, K. D. 2010. Efeito da qualidade da água no ciclo de vida e na atração para oviposição de Aedes aegypti (L.) (Diptera: Culicidae). Neotropical Entomology 39(6):1016-1023.

Beserra, E. B.; Santos-Dias, C. T. \& Parra, J. R. P. 2003. Características biológicas de linhagens de Trichogramma pretiosum desenvolvidas em ovos de Spodoptera frugiperda. Acta Scientiarum 25(2):479-483.

Blackmore, M. S. \& LoRd, C. C. 2000. The relationship between size and fecundity in Aedes albopictus. Journal of Vector Ecology 25:212-217.
CALAdO, D. C. \& NAVARRo-Silva, M. A. 2002. Influência da temperatura sobre a longevidade, fecundidade e atividade hematofágica de Aedes (Stegomyia) albopictus Skuse, 1894 (Diptera: Culicidae) sob condições de laboratório. Revista Brasileira de Entomologia 46(1):96-98

Choochote, W.; Tueton, B.; Kanjanapothi, D.; Rattanachanpichoi, E.; Chaithong, U.; Chainong, P.; JitPakdi, A.; TippawangKosol, P.; Rivong, D. \& Pitasawat, B. 2004. Potential of crude seed extract of celery, Apium graveolens L., against the mosquito Aedes aegypti (L.) (Diptera: Culicidae). Journal of Vector Ecology 29(2):340-346.

Delatte, H.; Gimonneau, G.; Triboire, A. \& Fontenille, D. 2009 Influence of temperature on immature development, survival, longevity, fecundity, and gonotrophic cycles of Aedes albopictus, vector of chikungunya and dengue in the Indian Ocean. Journal of Medical Entomology 43(1):33-41.

Fantinatti, E. C. S.; Duque, J. E. L.; Silva, A. M. \& Navarro-Silva, M. A. 2007. Abundância e agregação de ovos de Aedes aegypti L. e Aedes albopictus (Skuse) (Diptera: Culicidae) no norte e noroeste do Paraná. Neotropical Entomology 36(6):960-965.

Farnesi, L. C.; Martins, A. J.; Valle, D. \& Rezende, G. L. 2009. Embryonic development of Aedes aegypti (Diptera: Culicidae): influence of different constant temperatures. Memórias do Instituto Oswaldo Cruz 104(1): 124-126.

Gama, R. A.; Alves, K. C.; Martins, R. F.; Eiras, A. E. \& Resende, M. C. 2005. Efeito da densidade larval no tamanho de adultos de Aedes aegypti criados em condições de laboratório. Revista da Sociedade Brasileira de Medicina Tropical 34:64-66.

Joy, T. K.; Arik, A. J.; Corby-Harris, V.; Johnson, A. A. \& Riehle, M. A. 2010. The impact of larval and adult dietary on lifespan, reprodutction and growth in the mosquito Aedes aegypti. Experimental Gerontology 45:685-690.

Lowenberg Neto, P. \& Navarro-Silva, M. A. 2004. Development, longevity, gonotrophic cycle and oviposition of Aedes albopictus Skuse (Diptera: Culicidae) under cyclic temperatures. Neotropical Entomology 33(1):29-33.

Maciel-de-Freitas, R.; Eiras, A. E. \& Lourenço-De-Oliveira, R. 2008. Calculating the survival rate and estimated population density of gravid Aedes aegypti (Diptera: Culicidae) in Rio de Janeiro, Brazil. Caderno de Saúde Pública 24(12):2747-2754

MinistÉRIO DA SAÚDE. 2011. Sistema Nacional de Vigilância em Saúde, Relatório de Situação - Paraíba. 5 ed. Brasília, Ministério da Saúde. 35p.

Mohammed, A. \& Chadee, D. D. 2011. Effects of different temperature regimens on the development of Aedes aegypti (L.) (Diptera: Culicidae) mosquitoes. Acta Tropica 119:38-43.

Natal, D. 2002. Bioecologia do Aedes aegypti. Biológico 64(2):205207.

Ponlawat, A. \& Harrington, L. C. 2007. Age and body size influence male sperm capacity of the dengue vector, Aedes aegypti (Diptera: Culicidae). Journal of Medical Entomology 44:422-426.

Secretaria de Saúde do Estado da Paraíba. 2013. Boletim Epidemiológico $\mathbf{n}^{\circ}$ 05. João Pessoa, Governo do Estado da Paraíba. 6p.

Silva, H. H. G. \& SiLVA, I. G. 1999. Influência do período de quiescência dos ovos sobre o ciclo de vida de Aedes aegypti (Linnaeus, 1762) (Diptera, Culicidae) em condições de laboratório. Revista da Sociedade Brasileira de Medicina Tropical 32(4):349-355.

Tejerina, E. F.; Almeida, F. F. L. \& Almiron, W. R. 2009. Bionomics of Aedes aegypti subpopulations (Diptera: Culicidae) from Misiones Province, northeastern Argentina. Acta Tropica 109(1):45-49.

Vargas, R. E. M.; Ya-Umphan, P.; Phumala-Morales, N.; Komalamisra, N. \& Dujardin, J. 2010. Climate associated size and shape changes in Aedes aegypti (Diptera: Culicidae) populations from Thailand. Infection, Genetics and Evolution 10:580-585. 